Published in final edited form as:

Sleep Med. 2012 December ; 13(10): 1217-1225. doi:10.1016/j.sleep.2012.04.010.

\title{
Sleep Disturbances and Risk of Frailty and Mortality in Older Men
}

\author{
Kristine E. Ensrud, MD, MPH ${ }^{a, b, c,}{ }^{*}$, Terri L. Blackwell, MA ${ }^{\mathrm{d}, 1}$, Sonia Ancoli-Israel, $\mathbf{P h D}^{\mathrm{e}, 2}{ }^{\text {, }}$ \\ Susan Redline, MD', ${ }^{f}$, Peggy M. Cawthon, PhD ${ }^{\mathrm{d}, 1}$, Misti L. Paudel, MPH ${ }^{\mathrm{c}, 4}$, Thuy-Tien L. \\ Dam, MDg, ${ }^{\mathrm{g}}$, and Katie L. Stone, $\mathrm{PhD}^{\mathrm{d}, 1}$ for the Osteoporotic Fractures in Men (MrOS) Study \\ Group \\ ${ }^{a}$ Center for Chronic Disease Outcomes Research, Veterans Affairs Health Care System, \\ Minneapolis, MN \\ bDepartment of Medicine, University of Minnesota, Minneapolis, MN \\ 'Division of Epidemiology \& Community Health, University of Minnesota, Minneapolis, MN \\ ${ }^{d}$ Research Institute, California Pacific Medical Center, San Francisco, CA \\ eDepartment of Psychiatry, University of California - San Diego, La Jolla, CA \\ fDepartment of Medicine, Brigham \& Women's Hospital and Beth Israel Deaconess Medical \\ Center, Harvard Medical School, Boston, MA \\ gDepartment of Medicine, Columbia University, New York, NY
}

\section{Abstract}

Objective-To test the hypothesis that non-frail older men with poorer sleep at baseline are at increased risk for frailty and death at follow-up.

\begin{abstract}
Methods-In this prospective cohort study, subjective (questionnaires) and objective sleep parameters (actigraphy and in-home overnight polysomnography) were measured at baseline in 2,505 non-frail men aged $\ 67$ years. Repeat frailty status assessment was performed an average of 3.4 years later; vital status was assessed every four months. Sleep parameters were expressed as dichotomized predictors using clinical cut-points. Statuses at follow-up exam were classified as robust, intermediate (pre-frail) stage, frail, or died in interim.
\end{abstract}

\begin{abstract}
Results-None of the sleep disturbances were associated with the odds of being intermediate/ frail/dead (vs. robust) at follow-up. Poor subjective sleep quality (multivariable odds ratio [MOR] $1.26,95 \%$ CI 1.01-1.58), greater nighttime wakefulness (MOR 1.31, 95\% CI 1.04-1.66), and greater nocturnal hypoxemia (MOR 1.47, 95\% CI 1.02-2.10) were associated with a higher odds of frailty/death at follow-up (vs. robust/intermediate). Excessive daytime sleepiness (MOR 1.60,
\end{abstract}

\footnotetext{
*Center for Chronic Disease Outcomes Research, Minneapolis VA Health Care System, One Veterans Drive (111-0), Minneapolis, MN 55417, ensru001@umn.edu.

${ }_{2}$ SF Coordinating Center, 185 Berry Street, Lobby 5, Suite 5700, San Francisco, CA 94107

${ }_{2}^{2}$ Department of Psychiatry, University of California - San Diego, 9500 Gilman Drive 0733, La Jolla, CA 92063

${ }^{3}$ Brigham and Women's Hospital, Sleep Medicine Division, 221 Longwood Avenue, Boston, MA 02115

${ }_{5}^{4}$ Division of Epidemiology, University of Minnesota, 300 WBOB 7525, 1300 S 2nd St, Minneapolis, MN 55454

5 Department of Medicine, Allen Pavilion, Box 90 - Allen, New York, NY 10032

Disclosures

Drs. Ensrud and Stone have received grant support from the NIH (and supporting agencies) grant as listed above. Dr. Ancoli-Israel has received consultant fees or served on scientific advisory boards for Ferring Pharmaceuticals Inc., GlaxoSmithKline, Johnson \& Johnson, Merck, NeuroVigil, Inc., Pfizer, Philips, Purdue Pharma LP, Sanofi-Aventis, and Somaxon. All other authors have nothing to report.
} 
95\% CI 1.03-2.47), greater nighttime wakefulness (MOR 1.57, 95\% CI 1.12-2.20), severe sleep apnea (MOR 1.74, 95\% CI 1.04-2.89), and nocturnal hypoxemia (MOR 2.28, 95\% CI 1.45-3.58) were associated with higher odds of death (vs. robust/intermediate/frail at follow-up). The association between poor sleep efficiency and mortality nearly reached significance (MOR 1.48, 95\% CI 0.99-2.22). Short sleep duration and prolonged sleep latency were not associated with frailty/death or death at follow-up.

Conclusions-Among non-frail older men, poor subjective sleep quality, greater nighttime wakefulness, and greater nocturnal hypoxemia were independently associated with a higher odds of frailty or death at follow-up, while excessive daytime sleepiness, greater nighttime wakefulness, severe sleep apnea, and greater nocturnal hypoxemia were independently associated with an increased risk of mortality.

\section{Keywords}

sleep disturbances; frailty; mortality; men; elderly

\section{INTRODUCTION}

Self-reported and objectively measured sleep disturbances and frailty (a term indicating multisystem impairment and expanding vulnerability) are increasingly common with aging [1-6]. Previous studies in older adults have reported associations between sleep disturbances and individual components of frailty [6-10]. In addition, prior studies [9,11,12] have reported a greater prevalence of frailty among older adults with poor self-reported sleep quality, excessive daytime sleepiness, poor sleep efficiency, prolonged sleep latency, and sleep disordered breathing. However, all these previous studies utilized a cross-sectional design and the directionality of associations between sleep disturbances and frailty has not yet been evaluated with a prospective study.

Several prospective studies in older adults have reported that frailty is an independent predictor of increased mortality risk [1-3]. While previous studies have also examined the relationship between sleep disturbances and mortality in aged populations [13-21], findings are inconsistent and most have been limited to self-reported sleep measures. Thus, the longitudinal association of sleep disturbances among non-frail elders at baseline and risk of incident frailty and death is uncertain.

To test the hypothesis that non-frail older men with poorer sleep at baseline are at increased risk of frailty and death at follow-up, we measured subjective and objective sleep parameters in a cohort of 2,505 non-frail men aged 67 years and older at baseline enrolled in the Outcomes of Sleep Disorders in Older Men (MrOS Sleep) study, and reassessed frailty status and vital status an average of 3.4 years later.

\section{METHODS}

\section{Participants}

From March 2000 through April 2002, 5994 men $\searrow 65$ years of age were recruited for participation in the baseline examination of the prospective Osteoporotic Fractures in Men (MrOS) study [22]. Men were recruited from population based listings in six regions of the United States [23]. Men with a history of bilateral hip replacement and men who were unable to walk without the assistance of another person were excluded.

From December 2003 through March 2005, MrOS participants were invited to participate in an ancillary study to identify outcomes of sleep disorders in older men (MrOS Sleep study). A total of 3133 men (56\% of active survivors, $>100 \%$ of recruitment goal) completed the 
MrOS Sleep examination (baseline for this analysis) and provided adequate data for assessment of frailty status. Of these, a total of 2696 men who were classified as non-frail (robust or intermediate stage) at baseline were eligible for this longitudinal analysis. After excluding 191 surviving men missing repeat frailty status at the follow-up exam an average of 3.4 years later, the final analytical cohort was comprised of 2505 men including 2325 who had repeat assessment of frailty status at follow-up and 180 who died before the followup exam. The Institutional Review Board (IRB) at each center approved the study protocol and written informed consent was obtained from all subjects.

\section{Sleep Parameters}

Self-Reported Sleep Parameters-At the sleep examination, participants completed the Pittsburgh Sleep Quality Index (PSQI), a validated measure of subjective sleep quality and sleep disturbances over a one-month time period. Global PSQI scores range from 0-21. A score $>5$ is indicative of poor self-reported sleep quality and has a sensitivity of $89.6 \%$ and specificity of $86.5 \%$ in distinguishing good vs. poor sleepers [24].

Participants also completed the Epworth Sleepiness Scale (ESS), a self-administered questionnaire which classifies subjective daytime sleepiness among people with sleep disorders. Scores on the ESS range from 0-24, with a score $>10$ indicating excessive daytime sleepiness [25].

Objective Actigraphic Parameters of Sleep-Wake Patterns-As a part of the sleep examination, parameters of sleep-wake patterns were measured using an actigraph (Ambulatory Monitoring, Inc., Ardsley, NY), a small device used to detect movement that is similar in appearance to a wristwatch. Actigraphy has been shown to provide an objective and reliable estimate of sleep/wake patterns [26]. Actigraphy data were analyzed with ActionW- 2 software (Ambulatory Monitoring, Inc., Ardsley, NY). Details of the actigraphy scoring algorithms used in this study have been published elsewhere [27].

Participants were instructed to wear the actigraph continuously for five nights/six days, removing it only for bathing. They were also asked to keep a sleep log used to aid in editing the actigraph data. Actigraphy data was collected for an average of $5.2( \pm 0.9) 24$-hour periods.

Sleep-wake parameters examined in this analysis were actigraphy-based estimates of total sleep time (total hours slept while in bed), sleep efficiency (percentage of time participant was sleeping while in bed), sleep latency (amount of time until onset of sleep, defined as when participant achieved sleep for 20 continuous minutes after getting into bed), and time awake after sleep onset (defined as total minutes of time scored as awake from the onset of sleep until the end of the last sleep episode while in bed).

Objective Polysomnographic Parameters of Sleep Disordered Breathing-Inhome sleep studies were completed using unattended, portable polysomnography (Safiro ${ }^{\mathrm{TM}}$, Compumedics, Ltd., Abottsfield, AU). The recording montage was as follows: $\mathrm{C}_{3} / \mathrm{A}_{2}$ and $\mathrm{C}_{4} / \mathrm{A}_{1}$ electroencephalograms (EEG), bilateral electrooculograms and a bipolar submental electromyogram to determine sleep status; thoracic and abdominal respiratory inductance plethysmography to determine respiratory effort; airflow (by nasal-oral thermocouple and nasal pressure cannula); and finger pulse oximetry. Centrally-trained and certified staff performed home visits according to a protocol similar to that used in the Sleep Heart Health Study [28].

Parameters of sleep disordered breathing examined in this analysis included a measure of apneas/hypopneas, the apnea hypopnea index (AHI) and a measure of nocturnal hypoxemia, 
the percent of time during overnight sleep in which arterial oxygen saturation was below $90 \%$ (\% of sleep time with $\mathrm{SaO}_{2}<90 \%$ ). Apnea was defined as complete or near complete cessation of airflow for $>10$ seconds, and hypopneas were scored if clear reductions in breathing amplitude (at least 30\% below baseline breathing) occurred, and lasted $>10$ seconds [29]. In these analyses, only apneas and hypopneas that were each associated with a $4 \%$ or greater desaturation were included. AHI was calculated as the total number of apneas and hypopneas per hour of sleep.

\section{Other Measurements}

In addition to sleep measurements, participants completed a questionnaire and clinic visit including an interview and examination. A medical history was obtained by asking participants if they had ever received a physician diagnosis of selected medical conditions including prior fracture since age 50, arthritis, hypo/hyperthyroidism, diabetes mellitus, myocardial infarction, angina, stroke or temporary ischemic attack, claudication, congestive heart failure, chronic obstructive pulmonary disease (COPD), non-skin cancer, parkinsonism, chronic kidney disease or kidney failure, liver disease and hypertension. Participants were asked to bring all current medications used within the last 30 days with them to the sleep examination for verification of use. Physical activity was assessed using the Physical Activity Scale for the Elderly (PASE) [30]. Depressive symptoms were evaluated using the 15-item Geriatric Depression Scale [31]. Cognitive function was assessed with the Teng Modified Mini-Mental State Exam (3MS) [32]. Tests of physical function included grip strength (using a hand-held Jamar dynamometer) and walk speed (time in seconds to walk six meters at usual pace expressed as $\mathrm{m} / \mathrm{sec}$ ). Body weight and height measurements were used to calculate a standard body mass index (BMI).

\section{Treatment of Sleep Disordered Breathing}

After the sleep exam, participants were contacted every four months and asked about treatment for sleep disordered breathing, with response rates exceeding 99\%. A total of 156 men who reported starting a treatment (e.g., continuous positive airway pressure, supplemental oxygen, surgery for sleep disordered breathing, or use of a mouthpiece) between the sleep and follow-up examinations were excluded from the analyses examining the association of measures of sleep disordered breathing with frailty status and mortality outcomes.

\section{Frailty Status and Mortality}

Frailty status at the sleep exam (baseline for this analysis) was defined using criteria similar to those proposed by Fried and colleagues [2,3] using data collected in the Cardiovascular Health Study (CHS).

1. Shrinking as identified by unintentional weight loss of $25 \%$ between MrOS baseline and sleep examinations (mean years between examinations $3.4 \pm 0.5$ );

2. Weakness as identified by grip strength in the lowest quintile stratified by body mass index (quartiles);

3. Poor energy/exhaustion as identified by an answer of "no" to the question "Do you feel full of energy?" from the Geriatric Depression Scale (GDS);

4. Slowness as identified by walk speed in the lowest quintile stratified by standing height (median); and

5. Low physical activity level as identified by PASE score in the lowest quintile. 
Men with none of the above components were considered to be robust, those with one or two components were considered to be in an intermediate (pre-frail) stage, and those $\geq 3$ components were considered to be frail and excluded from this analysis.

Frailty status at the follow-up exam was defined using criteria cutpoints from the sleep exam. Since frailty and mortality are competing events, frailty status at follow-up and mortality between the sleep and follow-up exams were jointly analyzed by considering four outcome levels at follow-up: robust, intermediate stage, frail, or dead (died between sleep and follow-up exams). Vital status was ascertained by participant contacts every 4 months. Deaths were confirmed with death certificates. Follow-up for vital status was $99 \%$ complete.

\section{Statistical Analysis}

Differences in characteristics at baseline (sleep exam) according to frailty and mortality status at follow-up (robust, intermediate stage, frail, dead) were compared using analysis of variance for normally distributed continuous data, Kruskal-Wallis tests for skewed continuous data, and chi-square tests for categorical data.

For the analyses, the sleep parameter predictor variables were expressed as dichotomous variables based on published cutpoints for sleep disturbances, many of which define moderate to severe impairment (PSQI $>5$ vs. 5 , ESS $>10$ vs. 40 , total sleep time $<5$ hours vs. $\geq 5$ hours, sleep efficiency $<70 \%$ vs. $\geq 70 \%$, sleep latency $\geq 60$ minutes vs. $<60$ minutes, time awake after sleep onset $\geq 90$ minutes vs. $<90$ minutes, AHI $\geq 30$ vs. $<30$, and $\geq 10 \%$ of sleep time with $\mathrm{SaO}_{2}<90 \%$ vs. $<10 \%$ of sleep time with $\mathrm{SaO}_{2}<90 \%$ ). The prevalence of sleep disturbance at baseline (sleep exam) as defined by each parameter was compared across the four levels of the ordinal outcome at follow-up using the Cochran-Armitage test for trend.

The association between a given sleep disturbance and the ordinal outcome was initially examined using proportional odds models [33]. The assumption of homogeneity of effect of each dichotomous sleep predictor variable across all levels of the outcome was evaluated. However, the proportionality assumption was not met for all predictors. Thus, for consistency in presenting results, three separate logistic regression models were performed dichotomizing the outcome as intermediate stage/frail/dead (vs. robust), frail/dead (vs. robust/intermediate), and dead (vs. robust/intermediate/frail).

Initial base models were adjusted for age, clinic site, race, health status, and body mass index. Additional covariates were selected for inclusion in the final multivariable model if they were known correlates of sleep disturbances in the cohort or associated with follow-up status independent of age. Variables used to define each of the individual frailty components (such as physical activity) were not included in the covariate selection process. Covariates in the final multivariable models included age, race, site, health status, BMI, educational achievement, social support, alcohol intake, smoking status, antidepressant, benzodiazepine, nonbenzodiazepine sedative hypnotic use; number of comorbid medical conditions, cognitive function, and baseline frailty status (robust or intermediate stage).

In a secondary analysis to evaluate for evidence of a linear association between each sleep parameter and status at follow-up, the sleep measurements were expressed as continuous variables in base and final multivariable models. To graphically evaluate the presence of a linear associations between three continuous sleep parameters (time awake after sleep onset, the apnea hypopnea index and the percent of sleep time spent with $\mathrm{SaO} 2<90 \%$ ) and death, restricted cubic spline models were fit using knots specified at the $5^{\text {th }}, 25^{\text {th }}, 50^{\text {th }}, 75^{\text {th }}$, and $95^{\text {th }}$ percentile cutpoints. The cubic spline models were adjusted for age, race, site, health status and BMI (base model). To determine if there was evidence of a U-shaped relationship 
between total sleep time and follow-up status, secondary analyses also were conducted expressing total sleep time as a categorical predictor ( $\leq$ hours, $>5$ to 7 hours, $>7$ to $<8$ hours (reference) and $\geq 8$ hours). To evaluate the impact of including men classified as intermediate stage at baseline on the analyses examining associations of sleep disturbances with the odds of being intermediate/frail/dead at follow-up, we performed a sensitivity analyses for this outcome limiting the cohort to men classified as robust at baseline.

All significance levels reported were two-sided. All analyses were conducted using SAS version 9.2 (SAS Inc., Cary, NC).

\section{RESULTS}

\section{Characteristics of the Study Population}

Of the 2505 non-frail men (mean age 75.7 years) in the cohort, 941 (37.6\%) were classified as robust and $1564(62.4 \%)$ were in the intermediate stage at baseline. At the follow-up exam an average of 3.4 years later, $701(28.0 \%)$ were classified as robust, $1264(50.5 \%)$ were in the intermediate stage, $360(14.4 \%)$ were classified as frail, and $180(7.2 \%)$ had died in the interim period. Characteristics of the cohort according to frailty status and death at the follow-up exam are shown in Table 1. Among the 941 robust men at baseline, status at follow-up was robust in 468 (49.8\%), intermediate in 386 (41.0\%), frail in 50 (5.3\%), and died in interim in $37(3.9 \%)$. Among the 1564 men classified as intermediate stage at baseline, status at follow-up was robust in $233(14.9 \%)$, intermediate in $878(56.1 \%)$, frail in $310(19.8 \%)$, and died in interim in $143(9.2 \%)$.

Sleep disturbances at baseline were common in the cohort ranging from $8.9 \%$ for severe sleep apnea (AHI 230 ) up to $40.7 \%$ for poor self-reported sleep quality (PSQI >5) (Table 2). In unadjusted analyses there was evidence for a linear trend with a greater prevalence of each sleep disturbance at baseline with worsening status at follow-up ( $\mathrm{p} \unlhd \mathbf{\Delta} .01$ ), except for short sleep duration $(\mathrm{p}=0.06)$.

Compared with the longitudinal cohort of 2505 men, the 191 surviving men classified as non-frail at baseline who did not provide enough data for frailty status assessment at followup were, on average, slightly older ( 76.5 vs. 75.7 years, $\mathrm{p}=0.05$ ), but were no more likely to be classified as intermediate stage $(65.5 \%$ vs. $62.4 \%$, $\mathrm{p}=0.41)$.

\section{Longitudinal Associations of Self-Reported Sleep Disturbances with Frailty Status and Mortality}

In base models adjusted for age, race, health status and BMI, both self-reported poor sleep quality (PSQI >5) and excessive daytime sleepiness (ESS >10) were associated with a higher odds of being classified as intermediate/frail/dead (vs. robust) at follow-up (Table 3). However, these associations were attenuated and no longer significant after further adjustment for baseline frailty status (intermediate vs. robust). In final multivariate models adjusted for multiple potential confounders including baseline frailty status, poor sleep quality, but not excessive daytime sleepiness, was independently associated with higher odds of frailty or death (vs. robust/intermediate) at follow-up. In addition, excessive daytime sleepiness, but not poor sleep quality, was independently associated with a higher risk of death (vs. robust/intermediate/frail).

\section{Longitudinal Associations of Disruptions in Sleep-Wake Patterns with Frailty Status and Mortality}

Neither short sleep duration (total sleep time $<5$ hours) nor prolonged sleep latency (sleep latency $\succeq 60 \mathrm{~min}$ ) were associated with frailty status or death at follow-up (Table 4). In 
addition, neither poor sleep efficiency (sleep efficiency $<70 \%$ ) nor greater nighttime wakefulness (time awake after sleep onset $\geq 90 \mathrm{~min}$ ) were related to the odds of being intermediate/frail/dead at follow-up. There was some evidence of an independent association between poor sleep efficiency and an increased risk of mortality $(\mathrm{p}=0.054)$, but poor sleep efficiency was not related to the odds of frailty or death. Greater nighttime wakefulness was independently associated with higher odds of being frail or dead at follow-up and an increased risk of mortality.

\section{Longitudinal Associations of Sleep Disordered Breathing with Frailty Status and Mortality}

Neither severe sleep apnea (AHI 230 ) nor greater nocturnal hypoxemia ( $\geq 10 \%$ of sleep time with $\mathrm{SaO}_{2}<90 \%$ ) were associated with the odds of being intermediate/frail/dead at followup (Table 5). There was evidence of an independent association of greater nocturnal hypoxemia, but not severe sleep apnea, with a higher odds of frailty or death at follow-up. Both severe sleep and greater nocturnal hypoxemia were independently associated with an increased risk of mortality. Including a history of COPD as a specific covariate in multivariable modesl did not alter the associations of nocturnal hypoxemia and severe sleep apnea with these outcomes.

\section{Additional Analyses}

When the sleep parameters were expressed as continuous variables there was evidence to support linear independent associations of time awake after sleep onset, AHI, and percent of sleep time spent with $\mathrm{SaO} 2<90 \%$ with mortality (Figure 1). In contrast, there was no evidence of linear independent associations between any of the continuous sleep parameters and risk of frailty or death (see Supplementary Appendix). When total sleep time was expressed as a categorical variable, the lack of associations between sleep duration and frailty status and mortality at follow-up persisted. When the cohort was limited to men classified as robust at baseline, findings regarding the absence of independent associations between sleep disturbances and odds of being classified as intermediate/frail/dead at followup were not altered.

\section{DISCUSSION}

In this cohort of non-frail older men at baseline, self- reported poor sleep quality, objectively measured greater nighttime wakefulness, and greater nocturnal hypoxemia were associated with an increased risk of incident frailty or death at follow-up an average of 3.4 years later. The risk of mortality was higher among men with self-reported excessive daytime sleepiness, those with greater nighttime wakefulness, those with severe sleep apnea, and those with greater nocturnal hypoxemia.

Previous cross-sectional studies have reported independent associations between sleep disorders and individual components of frailty including exhaustion [7], weakness [8-10], slowness [8-10], and low physical activity [6]. In particular, a prior study of this cohort of older men [8] examining the association between sleep and physical function found slower gait speed and lower grip strength among men with poor sleep efficiency, those with greater nighttime wakefulness, and those with greater nocturnal hypoxemia. A previous study in this cohort [11] also reported independent associations between sleep disturbances (including self-reported poor sleep quality and objectively measured poor sleep efficiency, prolonged sleep latency, and sleep apnea [AHI 215$]$ ) and greater prevalent frailty status. In another study of older adults [6], an association between severe sleep apnea (AHI 230) and increased odds of prevalent frailty was observed among older women, but not men. Excessive daytime drowsiness, but not insomnia, was associated with higher odds of frailty in a study of community-living older persons [12]. 
This study with its prospective design examined whether sleep disturbances among nonfrail adults at baseline are associated with an increased risk of incident frailty and mortality at follow-up. Several previous studies examining the association between subjective sleep complaints and risk of death in older adults have reported inconsistent findings concerning the relationships of self-reported sleep duration and daytime sleepiness with mortality [1316]. In contrast to most of these studies, our investigation used validated survey instruments to assess self-reported sleep measures. The subsequent likelihood of frailty or death, but not mortality alone, was increased among men with poor sleep quality, while excessive daytime sleepiness was independently related to a higher risk of mortality, but not frailty or death. Although these results require confirmation, they suggest that, among self-reported sleep measures, sleep quality may have greater prognostic utility for assessing risk of frailty in older adults, while daytime sleepiness may have greater prognostic utility for assessing risk of mortality.

Additional studies have examined the association between objective measurements of sleep and risk of mortality in middle aged and older adults. Small studies have reported a Ushaped pattern between sleep duration as measured with actigraphy and survival at 10 years in postmenopausal women [17] and an increased risk of death among healthy elders with objectively measured prolonged sleep duration or poor sleep efficiency [18]. An association between sleep disordered breathing (as manifested by higher AHI or greater nocturnal hypoxemia) has been associated with an increased risk of mortality among middle-aged, but not older, adults [19-21]. In this cohort of community dwelling older men we found no evidence of associations between objective measures of sleep duration or prolonged sleep latency and risks of frailty or mortality. There was some evidence that men with poor sleep efficiency had a higher risk of death. Greater nighttime wakefulness and greater nocturnal hypoxemia were each independently associated with a higher risk of frailty or death, and greater nighttime wakefulness, severe sleep apnea (AHI 230 ), and greater nocturnal hypoxemia were each independently related to increased mortality. Findings regarding the association of greater nocturnal hypoxemia with these outcomes were not explained by the greater prevalence of a history of COPD among men with greater hypoxemia. These results suggest that, among objective measures of sleep, nighttime wakefulness and nocturnal hypoxemia may have prognostic utility in older adults for assessing both risks of frailty and death. There was evidence of linear independent associations between three measures (nighttime wakefulness, apnea hypopnea index, and nocturnal hypoxemia) and risk of mortality, but none of the sleep parameters expressed as continuous variables was independently related to the risk of frailty or death.

We found no evidence of independent associations between the presence of subjective or objective sleep disturbances at baseline and the odds of being intermediate/frail/dead (vs. robust) at follow-up. Taken in the context of our other findings, these results suggest that sleep disturbances may have greater prognostic utility for assessing the risk of frailty or death than assessing the risk of transitioning to a pre-frail state. The initial associations between poorer self-reported sleep measures and this outcome at follow-up were in large part due to the greater prevalence of intermediate stage or pre-frail state among those with poorer subjective sleep at baseline. Since the presence of the single poor energy component of frailty results in a classification of intermediate stage, this finding is not surprising.

The associations between sleep disturbances among non-frail older adults and the greater risks of frailty and mortality might be mediated through several mechanisms. Sleep disorders may be a marker of conditions including poor health, intermediate stage or prefrail state, and co-morbidities which, by themselves, impair sleep and increase the likelihood of frailty or death. However, these factors and other potential confounders explained some, but not all, of the associations observed between sleep disorders and incident frailty status. 
In addition, sleep disturbances have been linked to alterations in biochemical pathways including lower endogenous testosterone levels [34], renal dysfunction [35], and elevations in markers of chronic inflammation [36]. Any or a combination of these pathways might at least partially mediate the increased risk of frailty or death observed among men with sleep disorders. The association between sleep disturbances and frailty is also likely to be bidirectional in that frailty may lead to disruptions in rest activity rhythms with irregular sleep-wake cycles that are frequently observed in chronically ill older adults [12].

This study has a number of strengths, including large sample size, enrollment of communitydwelling older men not selected on the basis of sleep disorders or frailty status, comprehensive validated measures of frailty and subjective and objective sleep parameters, and prospective design. Analyses excluded men classified as frail at baseline and were adjusted for multiple potential confounders. Objective measurements of sleep-wake patterns were collected over multiple nights, resulting in more stable measures.

However, this study has several limitations. Participants were older predominantly Caucasian men, and findings might not apply to other groups. Measures used to define some frailty components were similar, but not identical, to those used in the original definition [2,3]. Analyses were adjusted for several factors, but the possibility of residual confounding cannot be eliminated. Surviving men not returning to the clinic for repeat assessment of frailty were slightly older at baseline, but were no more likely to be classified as pre-frail. Thus, it is unlikely that missing data markedly biased our findings. Analyses incorporated death as a level in the ordinal frailty status outcome because death is a competing event. Using this strategy, it is not possible to determine how much of the increased risks of frailty or death among men with greater nighttime wakefulness and those with greater nocturnal hxpoxemia are due to their higher risk of mortality. Participants did not have a clinical evaluation for primary sleep disorders such as restless leg syndrome and objective measures of obstructive lung disease using spirometry were not performed. All analyses were prespecified, but multiple comparisons were performed and some of our findings may be due to chance. Finally, average follow-up time in this study was 3.4 years, and it is uncertain whether the associations between sleep disturbances and incident frailty status persist or wane over the long-term.

Among non-frail older men, poor sleep quality, greater nighttime wakefulness, and greater nocturnal hypoxemia were associated with a higher risk of frailty or death at follow-up. Excessive daytime sleepiness, greater nighttime wakefulness, severe sleep apnea, and greater nocturnal hypoxemia were associated with an increased risk of mortality. Additional studies are warranted to evaluate the prognostic utility of sleep disturbances in predicting risk of frailty and death in older adults.

\section{Supplementary Material}

Refer to Web version on PubMed Central for supplementary material.

\section{Acknowledgments}

\section{Source of Funding}

The Osteoporotic Fractures in Men (MrOS) Study is supported by the National Institutes of Health funding. The following institutes provide support: the National Institute of Arthritis and Musculoskeletal and Skin Diseases (NIAMS), the National Institute on Aging (NIA), the National Cancer Institute (NCI), the National Center for Research Resources (NCRR), and the NIH Roadmap for Medical Research under the following grant numbers: U01 AR45580, U01 AR45614, U01 AR45632, U01 AR45647, U01 AR45654, U01 AR45583, U01 AG18197, U01AG027810, UL1 RR024140, and AG08415. 
The National Heart, Lung, and Blood Institute (NHLBI) provides funding for the MrOS Sleep ancillary study "Outcomes of Sleep Disorders in Older Men" under the following grant numbers: R01 HL071194, R01 HL070848, R01 HL070847, R01 HL070842, R01 HL070841, R01 HL070837, R01 HL070838, and R01 HL070839.

The funding agencies had no direct role in the conduct of the study; the collection, management, analyses, and interpretation of the data; or preparation or approval of the manuscript.

\section{Other Acknowledgments}

We would like to thank Mr. Kyle A. Moen for his assistance with the manuscript and preparation and formatting of the tables.

\section{References}

1. Cawthon PM, Marshall LM, Michael Y, Dam TT, Ensrud KE, Barrett-Connor E, et al. Frailty in older men: prevalence, progression, and relationship with mortality. J Am Geriatr Soc. 2007; 55(8): 1216-1223. [PubMed: 17661960]

2. Ensrud KE, Ewing SK, Cawthon PM, Fink HA, Taylor BC, Cauley JA, et al. A comparison of frailty indexes for the prediction of falls, disability, fractures, and mortality in older men. $\mathrm{J}$ Am Geriatr Soc. 2009; 57(3):492-498. [PubMed: 19245414]

3. Fried LP, Tangen CM, Walston J, Newman AB, Hirsch C, Gottdiener J, et al. Frailty in older adults: evidence for a phenotype. J Gerontol A Biol Sci Med Sci. 2001; 56(3):M146-M156. [PubMed: 11253156]

4. Mehra R, Stone KL, Blackwell T, Israel SA, Dam TT, Stefanick ML, et al. Prevalence and correlates of sleep-disordered breathing in older men: osteoporotic fractures in men sleep study. $\mathrm{J}$ Am Geriatr Soc. 2007; 55(9):1356-1364. [PubMed: 17767677]

5. Paudel ML, Taylor BC, Diem SJ, Stone KL, Ancoli-Israel S, Redline S, et al. Association between depressive symptoms and sleep disturbances in community-dwelling older men. J Am Geriatr Soc. 2008; 56(7):1228-1235. [PubMed: 18482297]

6. Young T, Shahar E, Nieto FJ, Redline S, Newman AB, Gottlieb DJ, et al. Predictors of sleepdisordered breathing in community-dwelling adults: the Sleep Heart Health Study. Arch Intern Med. 2002; 162(8):893-900. [PubMed: 11966340]

7. Goldman SE, Ancoli-Israel S, Boudreau R, Cauley JA, Hall M, Stone KL, et al. Sleep problems and associated daytime fatigue in community-dwelling older individuals. J Gerontol A Biol Sci Med Sci. 2008; 63(10):1069-1075. [PubMed: 18948557]

8. Dam TT, Ewing S, Ancoli-Israel S, Ensrud K, Redline S, Stone K. Association between sleep and physical function in older men: the osteoporotic fractures in men sleep study. J Am Geriatr Soc. 2008; 56(9):1665-1673. [PubMed: 18759758]

9. Endeshaw YW, Unruh ML, Kutner M, Newman AB, Bliwise DL. Sleep-disordered breathing and frailty in the Cardiovascular Health Study Cohort. Am J Epidemiol. 2009; 170(2):193-202. [PubMed: 19465743]

10. Goldman SE, Stone KL, Ancoli-Israel S, Blackwell T, Ewing SK, Boudreau R, et al. Poor sleep is associated with poorer physical performance and greater functional limitations in older women. Sleep. 2007; 30(10):1317-1324. [PubMed: 17969465]

11. Ensrud KE, Blackwell TL, Redline S, Ancoli-Israel S, Paudel ML, Cawthon PM, et al. Sleep disturbances and frailty status in older community-dwelling men. J Am Geriatr Soc. 2009; 57(11): 2085-2093. [PubMed: 19793160]

12. Vaz Fragoso CA, Gahbauer EA, Van Ness PH, Gill TM. Sleep-wake disturbances and frailty in community-living older persons. J Am Geriatr Soc. 2009; 57(11):2094-2100. [PubMed: 19793356]

13. Empana JP, Dauvilliers Y, Dartigues JF, Ritchie K, Gariepy J, Jouven X, et al. Excessive daytime sleepiness is an independent risk indicator for cardiovascular mortality in community-dwelling elderly: the three city study. Stroke. 2009; 40(4):1219-1224. [PubMed: 19246707]

14. Gangwisch JE, Heymsfield SB, Boden-Albala B, Buijs RM, Kreier F, Opler MG, et al. Sleep duration associated with mortality in elderly, but not middle-aged, adults in a large US sample. Sleep. 2008; 31(8):1087-1096. [PubMed: 18714780] 
15. Newman AB, Spiekerman CF, Enright P, Lefkowitz D, Manolio T, Reynolds CF, et al. Daytime sleepiness predicts mortality and cardiovascular disease in older adults. The Cardiovascular Health Study Research Group. J Am Geriatr Soc. 2000; 48(2):115-123. [PubMed: 10682939]

16. Stone KL, Ewing SK, Ancoli-Israel S, Ensrud KE, Redline S, Bauer DC, et al. Self-reported sleep and nap habits and risk of mortality in a large cohort of older women. J Am Geriatr Soc. 2009; 57(4):604-611. [PubMed: 19220560]

17. Kripke DF, Langer RD, Elliott JA, Klauber MR, Rex KM. Mortality related to actigraphic long and short sleep. Sleep Med. 2011; 12(1):28-33. [PubMed: 20870457]

18. Dew MA, Hoch CC, Buysse DJ, Monk TH, Begley AE, Houck PR, et al. Healthy older adults' sleep predicts all-cause mortality at 4 to 19 years of follow-up. Psychosom Med. 2003; 65(1):6373. [PubMed: 12554816]

19. Punjabi NM, Caffo BS, Goodwin JL, Gottlieb DJ, Newman AB, O’Connor GT, et al. Sleepdisordered breathing and mortality: a prospective cohort study. PLoS Med. 2009; 6(8):e1000132. [PubMed: 19688045]

20. Yaggi HK, Concato J, Kernan WN, Lichtman JH, Brass LM, Mohsenin V. Obstructive sleep apnea as a risk factor for stroke and death. N Engl J Med. 2005; 353(19):2034-2041. [PubMed: 16282178]

21. Young T, Finn L, Peppard PE, Szklo-Coxe M, Austin D, Nieto FJ, et al. Sleep disordered breathing and mortality: eighteen-year follow-up of the Wisconsin sleep cohort. Sleep. 2008; 31(8):10711078. [PubMed: 18714778]

22. Orwoll E, Blank JB, Barrett-Connor E, Cauley J, Cummings S, Ensrud K, et al. Design and baseline characteristics of the osteoporotic fractures in men (MrOS) study--a large observational study of the determinants of fracture in older men. Contemp Clin Trials. 2005; 26(5):569-585. [PubMed: 16084776]

23. Blank JB, Cawthon PM, Carrion-Petersen ML, Harper L, Johnson JP, Mitson E, et al. Overview of recruitment for the osteoporotic fractures in men study (MrOS). Contemp Clin Trials. 2005; 26(5): 557-568. [PubMed: 16085466]

24. Buysse DJ, Reynolds CF III, Monk TH, Berman SR, Kupfer DJ. The Pittsburgh Sleep Quality Index: a new instrument for psychiatric practice and research. Psychiatry Res. 1989; 28(2):193213. [PubMed: 2748771]

25. Johns MW. Reliability and factor analysis of the Epworth Sleepiness Scale. Sleep. 1992; 15(4): 376-381. [PubMed: 1519015]

26. Ancoli-Israel S, Cole R, Alessi C, Chambers M, Moorcroft W, Pollak CP. The role of actigraphy in the study of sleep and circadian rhythms. Sleep. 2003; 26(3):342-392. [PubMed: 12749557]

27. Blackwell T, Ancoli-Israel S, Gehrman PR, Schneider JL, Pedula KL, Stone KL. Actigraphy scoring reliability in the study of osteoporotic fractures. Sleep. 2005; 28(12):1599-1605. [PubMed: 16408420]

28. Redline S, Sanders MH, Lind BK, Quan SF, Iber C, Gottlieb DJ, et al. Methods for obtaining and analyzing unattended polysomnography data for a multicenter study. Sleep Heart Health Research Group. Sleep. 1998; 21(7):759-767. [PubMed: 11300121]

29. Quan SF, Howard BV, Iber C, Kiley JP, Nieto FJ, O'Connor GT, et al. The Sleep Heart Health Study: design, rationale, and methods. Sleep. 1997; 20(12):1077-1085. [PubMed: 9493915]

30. Washburn RA, Ficker JL. Physical Activity Scale for the Elderly (PASE): the relationship with activity measured by a portable accelerometer. J Sports Med Phys Fitness. 1999; 39(4):336-340. [PubMed: 10726435]

31. Sheikh JI, Yesavage JA. Geriatric depression scale (GDS): recent evidence and development of a shorter version. Clin Gerontol. 1986; 5(1/2):165-173.

32. Teng EL, Chui HC. The Modified Mini-Mental State (3MS) examination. J Clin Psychiatry. 1987; 48(8):314-318. [PubMed: 3611032]

33. Scott SC, Goldberg MS, Mayo NE. Statistical assessment of ordinal outcomes in comparative studies. J Clin Epidemiol. 1997; 50(1):45-55. [PubMed: 9048689]

34. Barrett-Connor E, Dam TT, Stone K, Harrison SL, Redline S, Orwoll E. The association of testosterone levels with overall sleep quality, sleep architecture, and sleep-disordered breathing. J Clin Endocrinol Metab. 2008; 93(7):2602-2609. [PubMed: 18413429] 
35. Canales MT, Taylor BC, Ishani A, Mehra R, Steffes M, Stone KL, et al. Reduced renal function and sleep-disordered breathing in community-dwelling elderly men. Sleep Med. 2008; 9(6):637645. [PubMed: 18819173]

36. Patel SR, Zhu X, Storfer-Isser A, Mehra R, Jenny NS, Tracy R, et al. Sleep duration and biomarkers of inflammation. Sleep. 2009; 32(2):200-204. [PubMed: 19238807] 
A

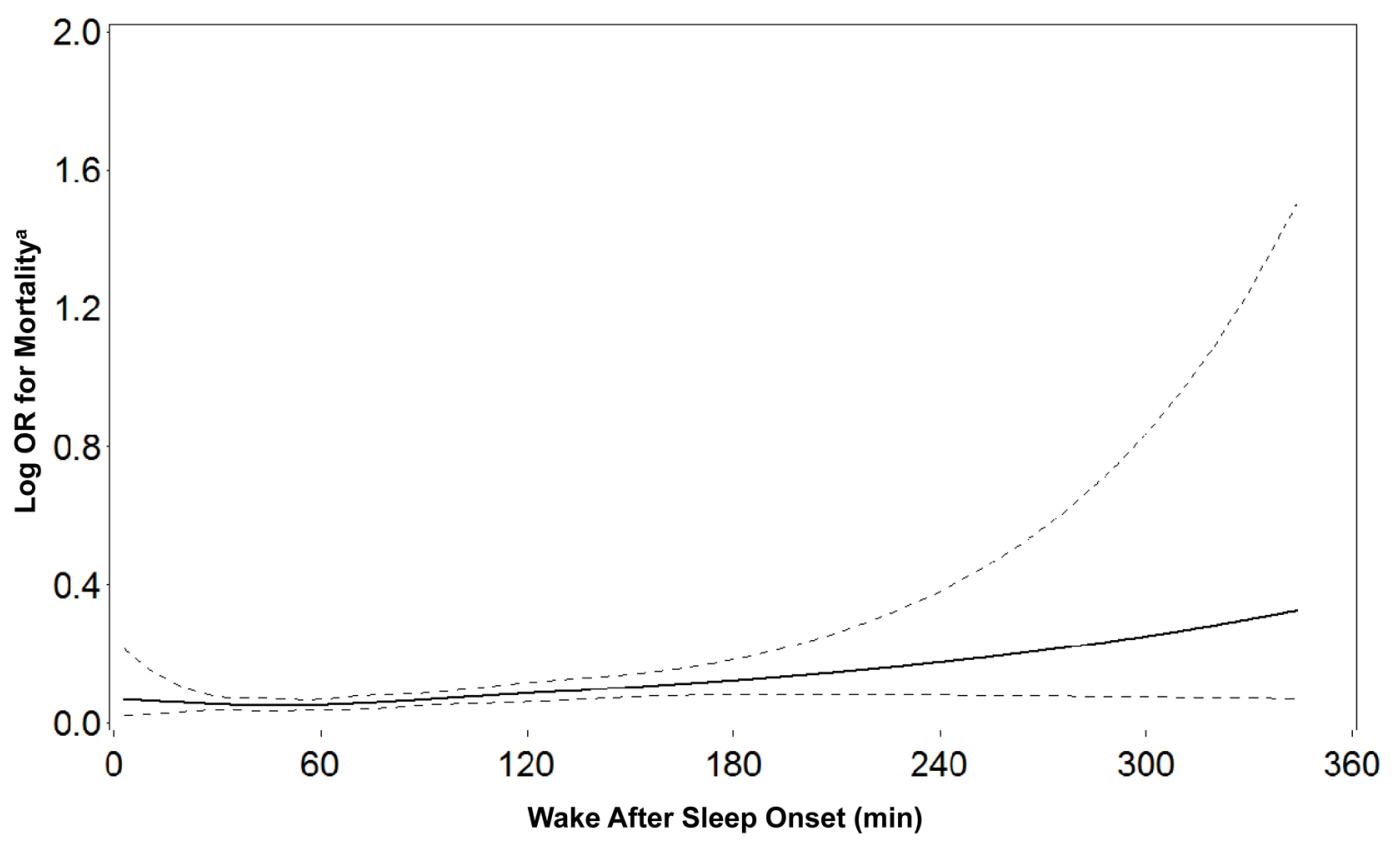

B

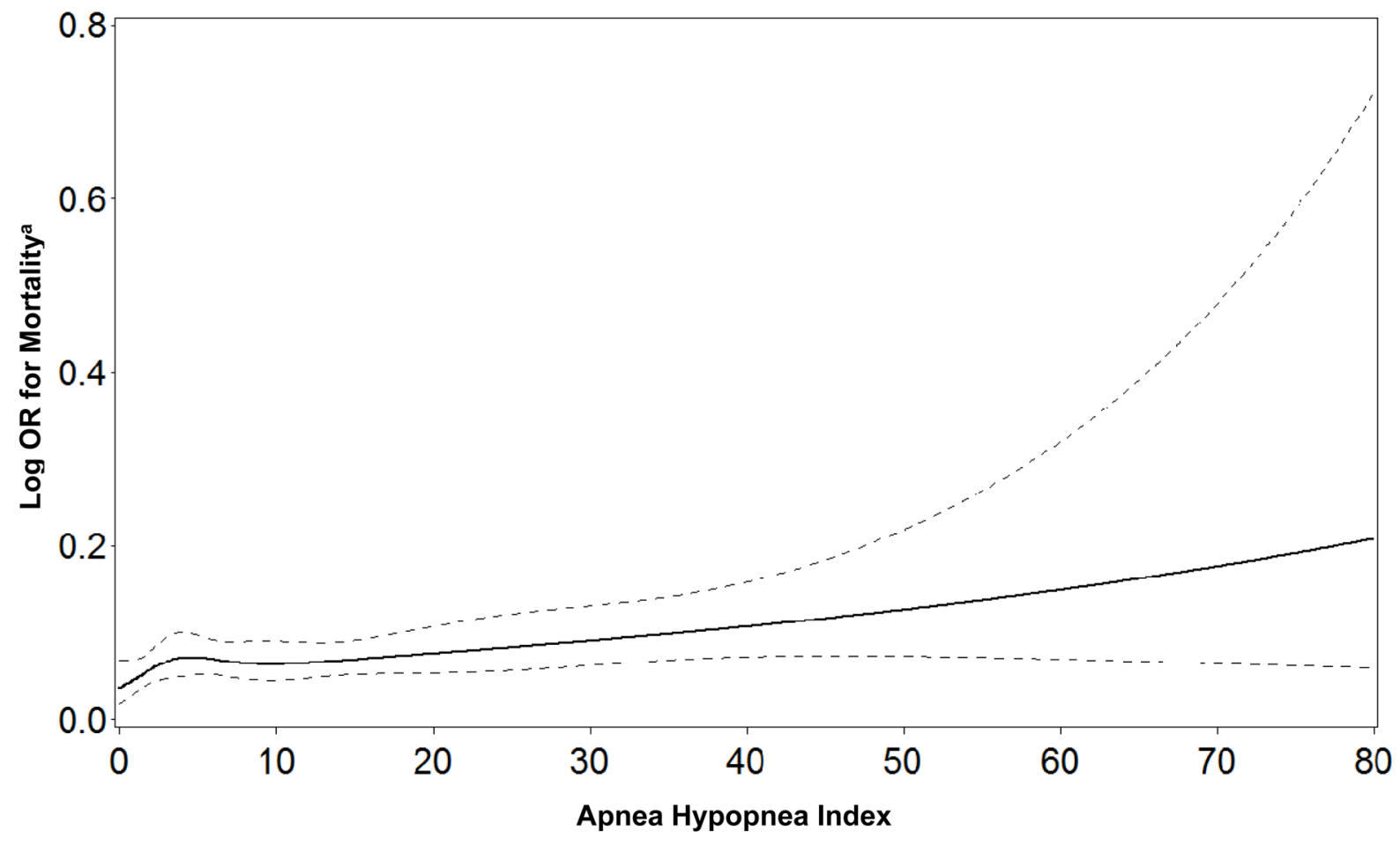


C

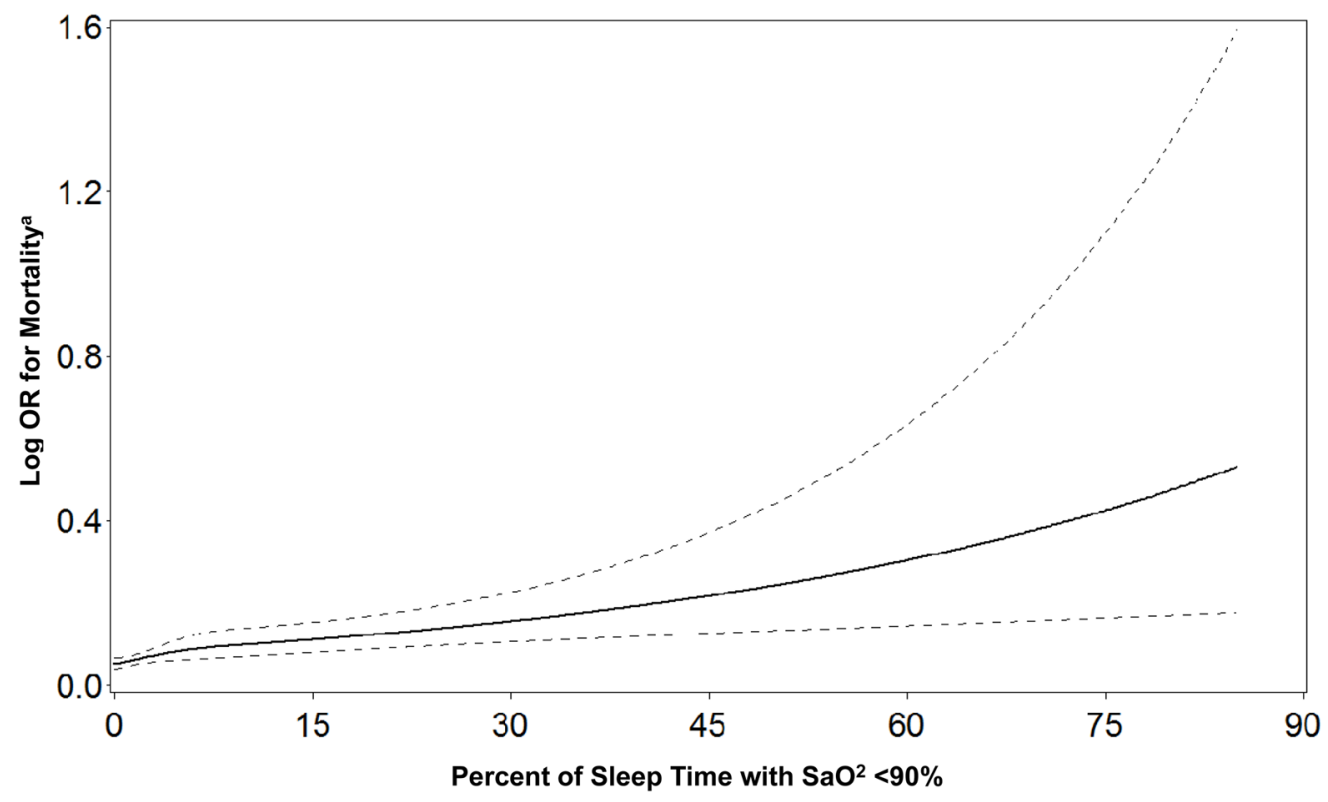

Figure 1.

Figure 1a-c. Restricted Cubic Spline Plots of Log Odds Ratio for Mortality by Level of Sleep Parameter

${ }^{a}$ Adjusted for age, race, site, health status, and body mass index 


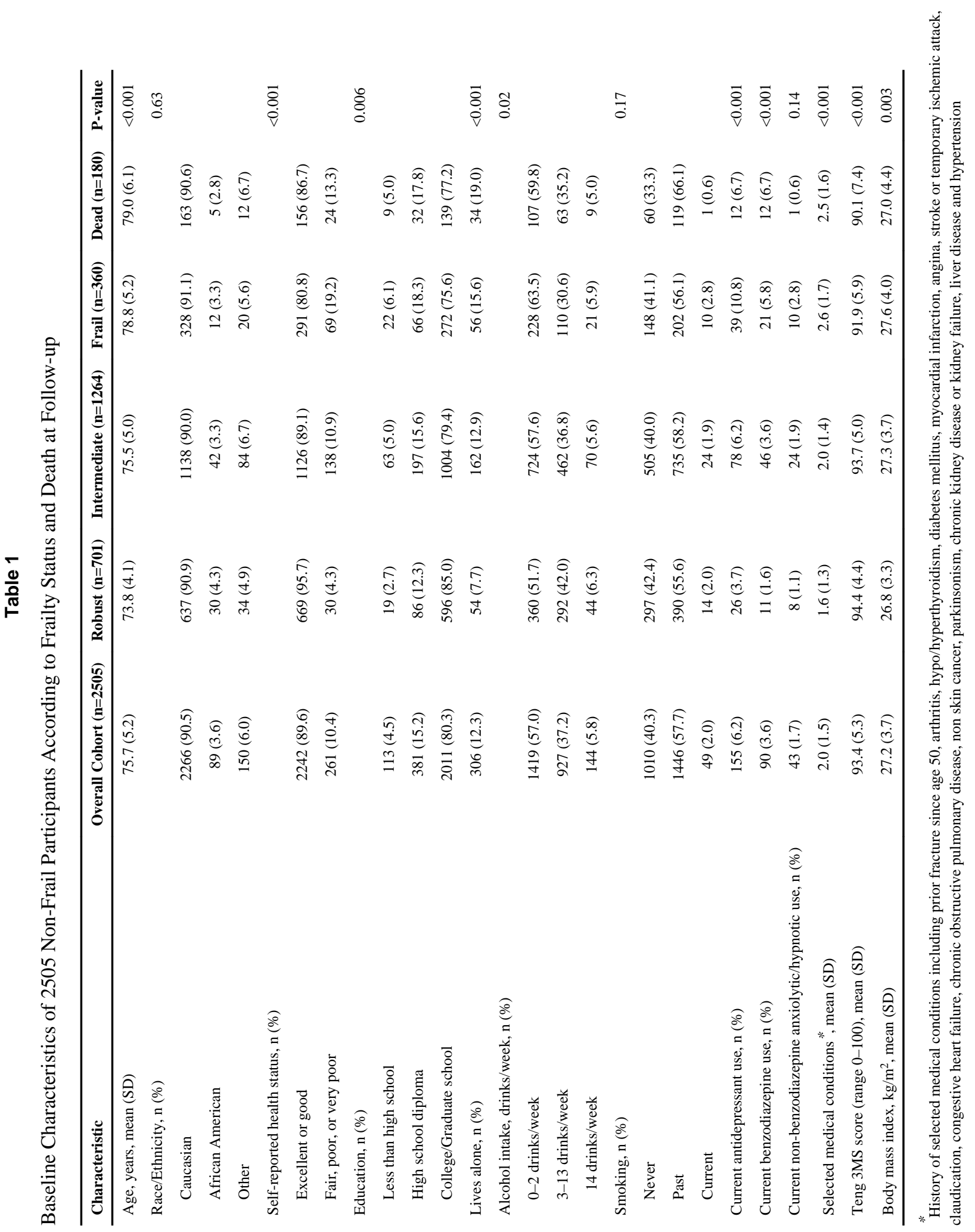




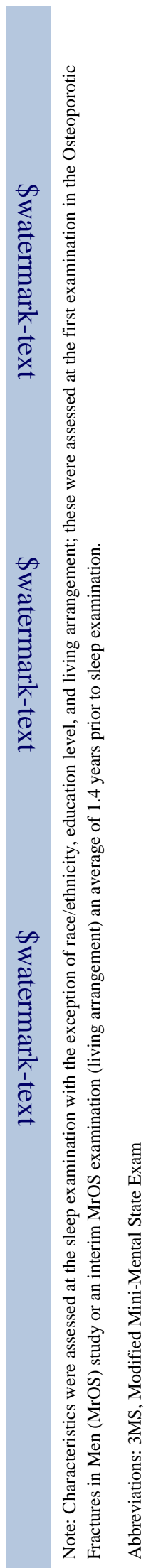

Sleep Med. Author manuscript; available in PMC 2013 December 01. 


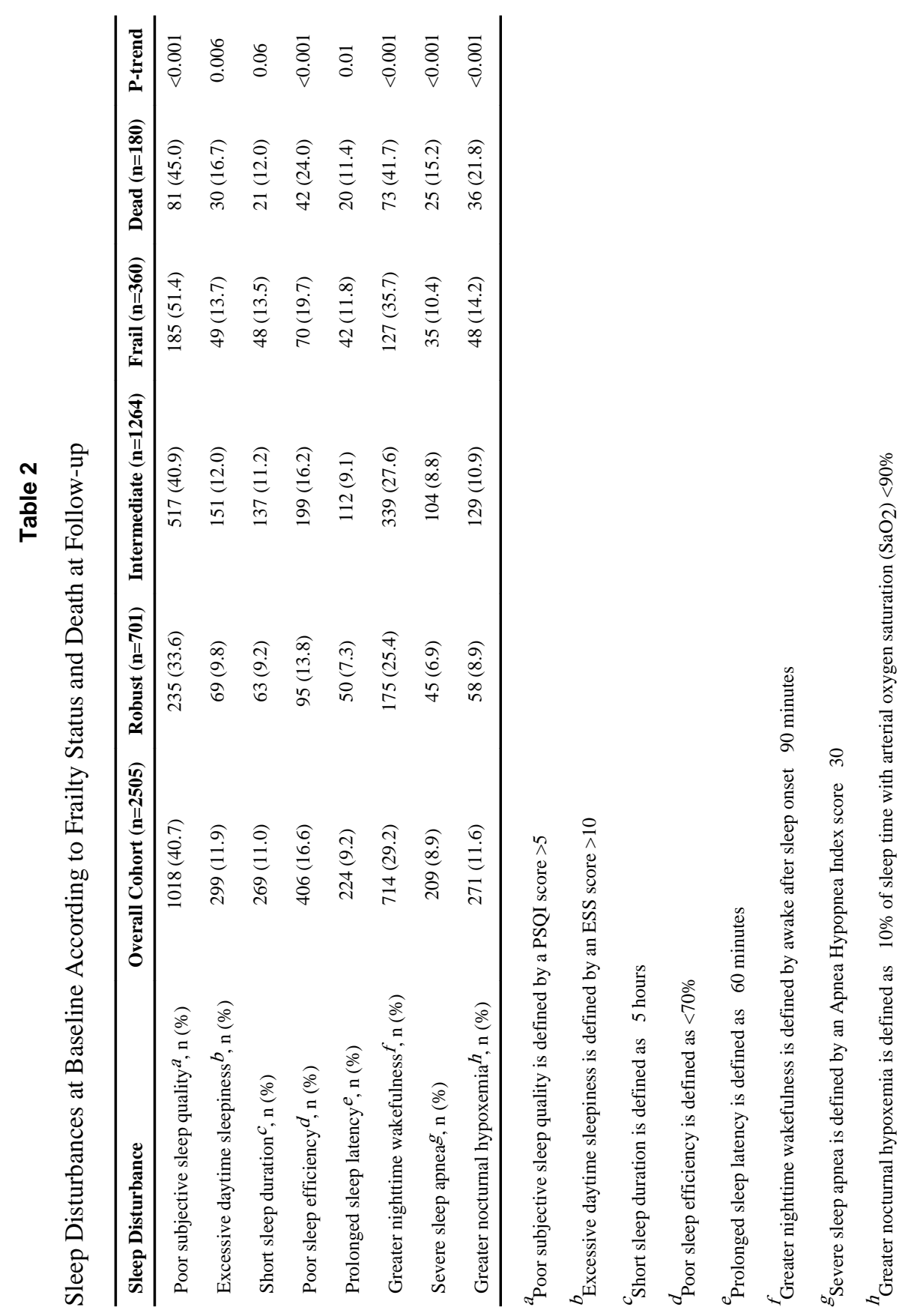




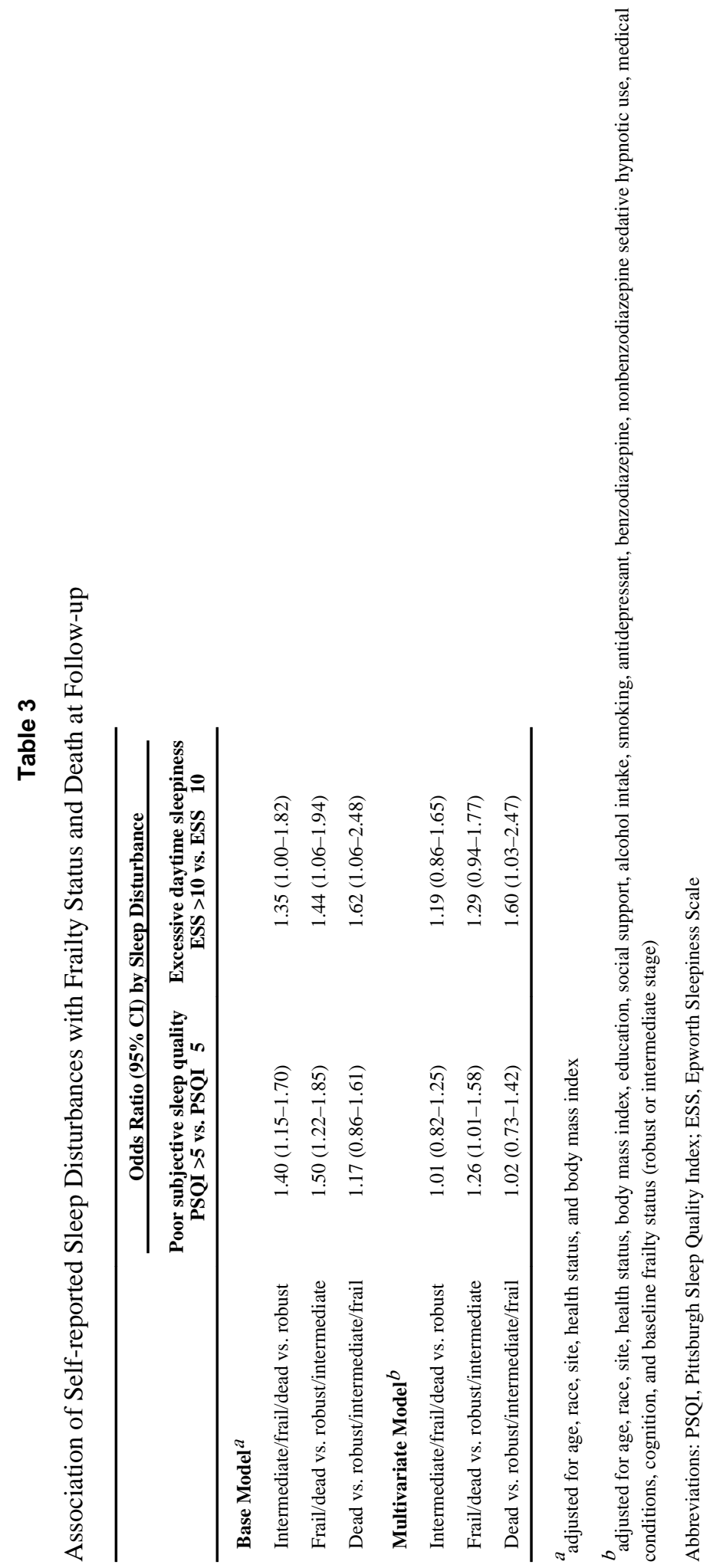




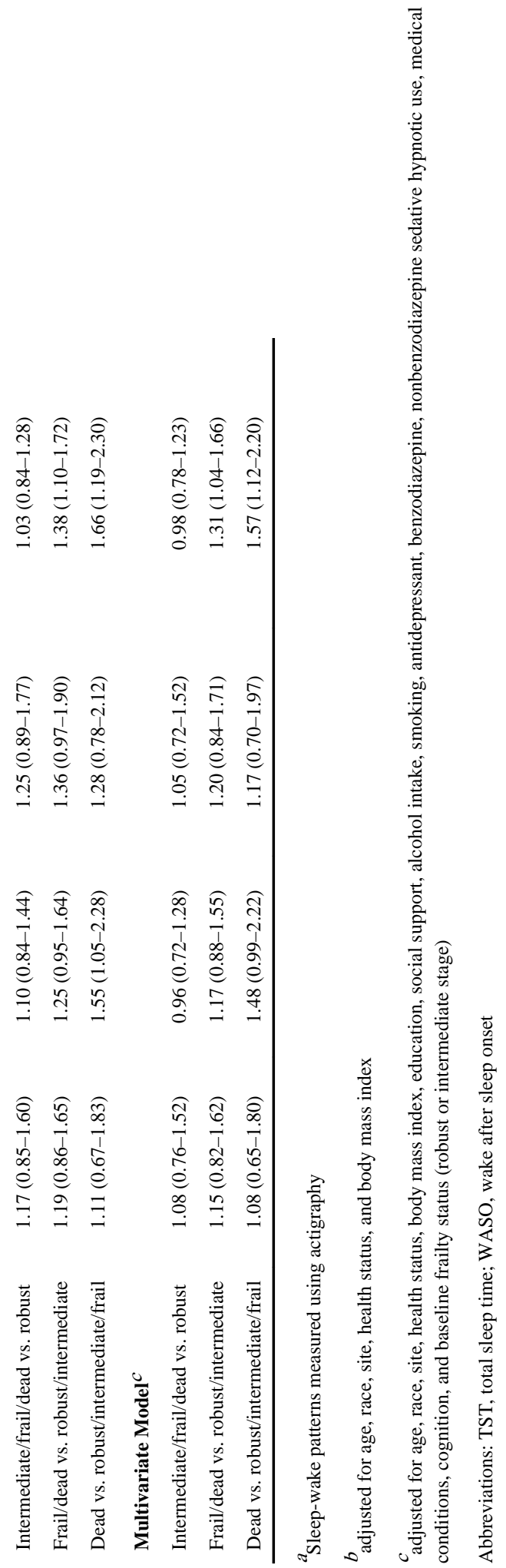


